\title{
Coverage Optimization Algorithm of Wireless Sensor Network Based on Mobile Nodes
}

\author{
http://dx.doi.org/10.3991/ijoe.v12i08.6068 \\ Li Zhu ${ }^{1}$, Chunxiao Fan ${ }^{1 *}$, Huarui $\mathrm{Wu}^{2,3,4}$ and Zhigang $\mathrm{Wen}^{1}$ \\ ${ }^{1}$ Beijing University of Posts and Telecommunications, Beijing, China \\ ${ }^{2}$ Beijing Research Center for Information Technology in Agriculture, Beijing, China \\ ${ }^{3}$ National Engineering Research Center for Information Technology in Agriculture, Beijing, China \\ ${ }^{4}$ Key Laboratories for Information Technology in Agriculture, Beijing, China
}

\begin{abstract}
To reduce the blind zone in network coverage, we propose a coverage optimization algorithm of wireless sensor network based on mobile nodes. This algorithm calculates the irregularity of blind zone in network coverage and obtains the minimum approximate numerical solution by utilizing the quantitative relationship between energy consumption of related nodes and the position of the mobile nodes. After determining the optimal relative position of the mobile nodes, the problem of blind zone between the static nodes is addressed. Simulation result shows that the proposed algorithm has high dynamic adaptability and can address the problem of blind zone maximally. Besides increasing the network coverage, the algorithm also reduces the network energy consumption, optimizes network coverage control and exhibits high convergence.
\end{abstract}

Index Terms-mobile node; Wireless Sensor Network; network coverage rate; static nodes; the blind zone.

\section{INTRODUCTION}

Wireless sensor network consists of a large number of sensor nodes, which are randomly deployed within a certain monitoring region. The nodes collaborate in obtaining data of different environmental factors. Now wireless sensor network has been widely applied in the fields of medicine, agriculture, military and environmental monitoring. Because wireless sensor networks are composed of large number of inexpensive wireless sensor nodes, the finite energy has become one of the key factors that restrict the performance of the network. Taking into account the wireless sensor networks are usually deployed in relatively poor place, more difficult to replace the battery or change.Coverage is an important indicator of wireless sensor network [1-3] and optimizing coverage is to reduce the blind zone and redundant coverage, to ensure high data transmission efficiency and to prolong the service life within an acceptable range of energy consumption In order to improve the performance of WSN, and cover the requirements for monitoring area, the densely deployment is one of the most common method to improve the coverage rate. But in this deployment strategy, there are large number of redundant nodes, and bring some energy consumption, such as the data redundancy acquisition, wireless channel interference, and worsen channel conflict, although the coverage is guaranteed. The network node density control is proposed in the premise of not affecting the network performance, combined with the current network running status, and regulate part of the redundant nodes to sleep state, aim to reduce the redundancy net- works, reduce the network energy consumption, and extend the network lifetime etc.

The coverage rate is an important parameter of WSN obtain integrity and nonrepudiation information. This reflects WSN perception ability, which was used to describe the quality of service. In order to achieve full coverage of the monitor area, the most usual way to improve the coverage rate of wireless sensor network is dense deployment. In this deployment strategy, coverage rate was assured, but there is perception coverage intersection between nodes in the network, which will cause some problems such as information data redundancy acquisition, channel conflict and so on. Meanwhile, input-output ratio was considered in the environmental monitoring, so the dense deployment strategy is not usually adopted when the fund is insufficient.

Some major achievements have been made in wireless sensor network coverage. Tian et al. propose off-duty eligibility rule [4], which calculates the coverage relations of the nodes based on node position or angle of arrival. But this algorithm does not consider the risk of redundant coverage between the nodes, leading to many overlapping regions of the network, necessitating too many worker nodes and causing high energy consumption. Bulusu $\mathrm{N}$ utilized self-adaptive node deployment algorithm based on radio beacons [5], which repairs the voids by adding radio beacons. But no consideration was given to the cost of adding radio beacons and the impact on the monitored environment. The literature [6] proposed an adaptive node scheduling algorithm, the algorithm uses the mechanism of message passing between nodes for the active node monitoring area information, through the adaptive mechanism to adjust the working mode of all nodes, the redundant nodes in a certain period of time, in the sleep mode to reduce the network energy costs, but as a result of information acquisition by that constantly sending messages between the nodes, make some nodes to undertake large amount of work tasks, appearing premature "death" phenomenon, affects the whole network connectivity and overall performance. Literature [7] employed grids for detecting voids and made simplifications of the procedures by assuming that the uncovered grids are located within the voids. But the algorithm may fail to detect triangular voids sometimes. Wang [8] focused on the impact of data fusion on sensor network coverage. The collaboration of the nodes was analyzed using probabilistic sensor model based on power attenuation, and the energy-efficient collaboration detection scheme was de- 
scribed with the consideration of the balance between coverage and energy consumption. However, the position of the sensor nodes relative to the target was not considered with respect to its contribution to the coverage. Literature [9] put forward using sensor nodes perception range adjustable, establish Delaunay triangle between the adjacent nodes, find out Delaunay triangle remaining power center of gravity, the algorithm only considers the coverage and network distribution of node, don't compare the node remaining power, reduce the network lifetime, which have some limitations.Cheng [10] proposed a distributed greedy algorithm for enhancing probabilistic coverage, where the sensing direction of each node is set according to a priority sequence and the sensing direction is adjusted once at the most for each node. Ma [11] devised an adaptive triangular deployment scheme, which divides the monitoring region into fan-shaped zones. To reduce blind zones, the node deployment is optimized within the fanshaped zones. But the equilibrating movement of nodes, movement distance minimization and network connectivity are omitted. Literature [12] is an integration of two network coverage control algorithms. Several primary nodes are selected randomly as the centers to define a polygonal zone, which is followed by addressing the blind zones at a fixed distance near the primary nodes. This is a more mechanical method for addressing the problem of blind zones and may lead to overlapping of coverage. This study introduces a coverage optimization algorithm of wireless sensor network based on mobile nodes. For static nodes and dynamic modes, different geometric relations between them are quantified based on the position of blind zones and related nodes. Then mobile nodes are used and the mobile node optimization model is established with the consideration of sensing scope and residual energy. Blind zones are repaired according to the quantitative relationship between energy consumption of related nodes and the position of the mobile nodes. With the minimum approximate numerical solution obtained $[13,14]$, the optimal position of mobile nodes and the number of mobile nodes needed are calculated for the purpose of coverage maximization. This not only resolves the problem of overlapping related to repairing the static voids, but also reduces the number of nodes and prolongs the service life.

\section{NETWORK MODEL AND PROBLEM DESCRIPTION}

\section{A. Network model}

$\mathrm{N}$ nodes of wireless sensor networks are randomly and evenly deployed within a $2 \mathrm{D}$ monitoring area $\mathrm{A}$, which is supposed to have the following properties:

(1) Probabilistic sensor model is used for the nodes;

(2) Node communication radius $R_{c}$ and the maximum sensing radius of the node has $R_{c} \geq 2 R_{m}$ relation; any two nodes located at a distance less than the communication radius are considered neighbor nodes.

(3) The nodes are either static or mobile in the network. The initial energy of all nodes is $\mathrm{W}$ and the nodes are synchronized.

(4) The precise position of the nodes in the network is known.

(5) The static nodes are connected and the problem of network connectivity is not considered when optimizing network coverage.

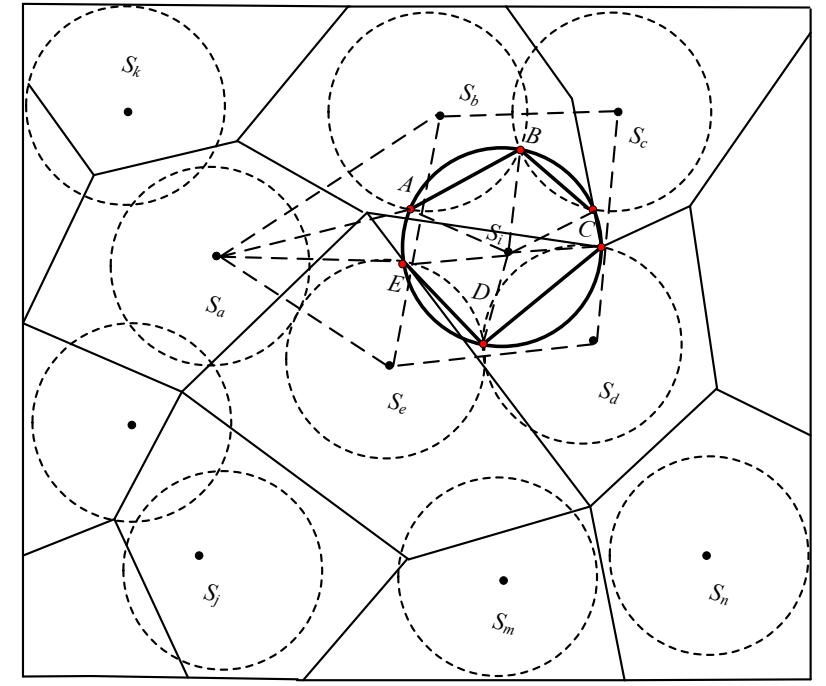

Figure 1. position of the mobile nodes

\section{B. Problem description}

Any nodes in the network can acquire the information of themselves and their neighbors. Every node has a unique ID and it acquires the attribute information of the neighbor nodes during communication. As mentioned above, when $R_{c} \geq 2 R_{S}$, the neighbor nodes are well connected. When the monitoring region is completely covered by the network, the network nodes must be connected, and vice versa. The blind zones in the network are irregular polygons [15-17]. As shown in the figure 1, for neighbor nodes $\mathrm{Sa}, \mathrm{Sb}, \mathrm{Sc}, \mathrm{Sd}$ and $\mathrm{Se}$, there is a blind zone M. A node set $\mathrm{B}$ is configured for $\mathrm{M}$ for completely repairing the blind zone. Then before the repair, the area $M$ $S_{M}=S_{B}-\sum_{i=a}^{e}\left(S_{B} \mathrm{I} S_{i}\right)$ . Within this range, mobile nodes are introduced for the repair based on the following procedures: (1) determine the position between the mobile nodes and the related neighbor nodes in $\mathrm{M}$; (2) introduce the mobile nodes such that the area to be repaired is minimized without increasing new blind zone; (3) reduce redundant coverage caused by random deployment. We propose a coverage optimization algorithm of wireless sensor network based on mobile nodes. Under the premise that the blind zones are known, the positions of blind zones and related nodes are analyzed and the mobile node optimization model is built considering the factors of sensing scope and residual energy. By quantifying the relationship between energy consumption of related nodes and position of the mobile nodes, redundant coverage is addressed later and the minimum approximate numerical solution is obtained. Therefore, network coverage is maximization based on mobile nodes.

\section{COVERAGE OPTIMIZATION STRATEGY}

\section{A. Definitions}

Definition 1 (related nodes in blind zone): There are several polygonal blind zones of varying size and shapes in the monitoring region. These blind zones are not covered by any nodes of the network. When the distance from the sensor node Si to any side of the boundary of the blind zone is smaller than 2 times the sensing radius $\mathrm{R}_{\mathrm{s}}$, node $\mathrm{Si}$ is called the related node of the blind zone. 
Definition 2 (independent blind zone): If zone A is not covered by any node in the network and there are no related nodes along its boundaries, then zone $\mathrm{A}$ is considered the independent blind zone.

Definition 3 (closed blind zone): If zone A is not covered by any node in the network, but there is at least 1 related node along its boundaries, then zone A is considered the closed blind zone.

Definition $4 \square$ cover point $\square \operatorname{Cov}(i)$ represents that the target point $\mathrm{i}$ was covered, namely

$$
\operatorname{Cov}(i)= \begin{cases}1 & \Phi(i) \geq \varepsilon \\ 0 & \Phi(i)<\varepsilon\end{cases}
$$

$\Phi(i)$ is perceived strength of $i, \varepsilon$ is perception threshold.

Definition $5 \square$ cover node subset $\square$ sensor nodes set $\mathrm{S}$ were deployed in monitoring area $\mathrm{A}$, that is $\mathrm{S}=\left\{\mathrm{S}_{1} \ldots \ldots \mathrm{S}_{\mathrm{i}} \ldots \ldots \mathrm{s}_{\mathrm{n}}\right\}$, D is the subset of $\mathrm{A}$, Sensor node set $\mathrm{D} \subseteq \mathrm{S}$, if any node in $\mathrm{V}$ can cover monitoring area $\mathrm{D}$ completely, $\mathrm{V}$ is cover node subset of $\mathrm{S}$.

Definition 6 (covered zone): Consider any target region A. If the presence of any point $\mathrm{k}$ in the target region is sensed by node $i$, then this point is said to be covered by node $i$. If all nodes in the target region are covered by node $i$, then this region is called the covered zone $M_{i}$ of node $i$. Here the covered zone of node $i$ is the intersection of covered zone $\mathrm{M}_{\mathrm{i}}$ and the area $\mathrm{S}_{\mathrm{A}}$ of the target region $\mathrm{A}$ or target node $\mathrm{k}$ and is denoted as $\Phi_{\mathrm{i}}$.

$$
\Phi_{i}=\left(\mathrm{M}_{i} \mathrm{I} S_{A}\right) \mathrm{U}\left(\mathrm{M}_{i} \mathrm{I} \sum_{k=1}^{n} S_{k}\right)
$$

Definition 7:( Coverage Blind zones) In any polygon, $\Phi(i)$ is the Coverage Blind zones of the node $i$ .Supposing the target monitoring region $\Omega$ is included in the polygon of node $i$, and $\Omega$ is not perceived by node $i$. Then $\Phi(\mathrm{i})$ can be expressed as below.

$$
\Phi(\mathrm{i})=\{\Omega \subset \mathrm{V}(\mathrm{i}) \mid \Omega \mathrm{I} \Psi(\mathrm{i})=\phi\}
$$

Definition 8 (coverage rate): For the covered zone of node $\xi=\pi R_{s}^{2}$, when and only when any node $\mathrm{p}$ in the monitoring region $\left\|S_{i} \cdot p\right\| \leqslant R_{s}$, node $\mathrm{p}$ is said to be covered by $\mathrm{S}_{\mathrm{i}}$. Coverage $\eta$ is the ratio of covered zone of all operating nodes in region $\mathrm{A}$ to the total monitoring region.

$$
\eta=\frac{\sum_{i=1}^{n} \xi(i)}{A}
$$

Definition $9 \square$ coverage balance degree $\square$ coverage balance degree can reduce the network energy consumption, avoid the failure of some nodes by balance work task and energy of nodes.

$$
I=\sum_{i=1}^{n} \sqrt{\frac{1}{m_{i}} \sum_{j=1}^{m_{i}}\left[d(i, j)-h_{i}\right]^{2}} / N
$$

In formula (5) represents the coverage balance degree, $\mathrm{N}$ is the total number of the network nodes, $\mathrm{d}(\mathrm{i}, \mathrm{j})$ is the
Euclidean distance between nodes $\mathrm{i}$ and $\mathrm{j}$, the neighbor nodes number of $\mathrm{I}$ is $\mathrm{mi}, \mathrm{h}_{\mathrm{i}}$ is the average of the distancesaid between node I and $\mathrm{k}$, the perceive area of node $\mathrm{k}$ and $\mathrm{i}$ were overlap.

\section{B. Coverage optimization strategy based on mobile nodes}

Coverage blind zones are composed of points or areas not covered by the network nodes. They are not sensed or covered by any nodes [18]. The irregularity of the blind zones is analyzed by using the mobile nodes. The minimum approximate numerical solution is calculated according to the quantitative relationship between energy consumption of related nodes in the blind zone and the position of mobile nodes. Then network coverage is maximized by determining the optimal position of the mobile nodes and the number of mobile nodes needed. On this basis, the blind zones can be repaired.

Suppose the monitoring region $\mathrm{M}$ is composed of $\mathrm{N}$ randomly distributed sensor nodes. For any node $S_{k}$ and the set $H$ of its neighbor nodes, the area $\Omega$ of the irregular blind zone is calculated according to the relative position of node $S_{k}$ and its neighbor nodes. Fig. 2 shows the closed and the independent blind zones. The area of irregular polygonal blind zone is calculated from the Euclidean distance and the distribution of the covered zones. For sensor node $\mathrm{S}_{\mathrm{k}}$, its sensing radius $\mathrm{R}_{\mathrm{s}} \in\left[\mathrm{R}_{0}, \mathrm{R}_{\mathrm{m}}\right]$ and its maximum covered area $\mathrm{E}\left(\mathrm{S}_{\mathrm{k}}\right)=\pi \mathrm{R}_{\mathrm{m}}{ }^{2}$. If the blind zone is closed and has $m$ related nodes without redundant coverage, then the total maximum covered area $\Psi=\bigcup_{\mathrm{k}=1}^{\mathrm{m}} \mathrm{E}\left(\mathrm{S}_{\mathrm{k}}\right)$. But considering the overlaps between the covered zones, the total covered area of $m$ related nodes is $\Lambda \leq \bigcup_{\mathrm{k}=1}^{\mathrm{m}} \mathrm{E}\left(\mathrm{S}_{\mathrm{k}}\right)$. For any node $S_{k}$ in the monitoring region, the connections with its neighbor nodes constitute the minimal triangular network. $h_{k}$ is the boundary of sensing of node $S_{k}$. The sensing areas of node $S_{k}$ and $S 0$ intersect, and the coverage arc corresponding to the overlapping boundary is $\mathrm{L}_{\mathrm{k} 0}$. $\mathrm{L}_{\mathrm{k} 0}=\mathrm{h}_{\mathrm{k}} \mathrm{I} \mathrm{h}_{0}$ The coverage angle corresponding to the coverage arc is $\alpha_{\mathrm{k} 0}$. When node $\mathrm{S}_{\mathrm{k}}$ is located in any position within the monitoring region, the set of its neighbor nodes is $\mathrm{H}\left\{\mathrm{S}_{0} \ldots \mathrm{S}_{\mathrm{j}} \ldots \mathrm{S}_{\mathrm{n}}\right\}$. Using the relationship between the sensing scope and the distance between the neighbor nodes, the area $\Omega\left(S_{k}\right)$ of the blind zone formed by node $S_{k}$ and its neighbor node is approximately given by

$$
\Omega\left(\mathrm{S}_{\mathrm{k}}\right)=\Theta\left(\mathrm{US}_{\mathrm{j}=1}^{\mathrm{n}}\right)-\sum_{\mathrm{j}=1}^{\mathrm{n}} \pi \mathrm{r}_{\mathrm{j}}^{2} \cdot \Gamma\left(\mathrm{L}_{\mathrm{kj}}, \alpha_{\mathrm{kj}}\right) \cdot \mathrm{d}\left(\mathrm{S}_{\mathrm{k}}, \mathrm{S}_{\mathrm{j}}\right)
$$

where $\Theta\left(\bigcup_{j=1}^{n} S_{j}\right)$ is the total area of boundary regions of node $\mathrm{S}_{\mathrm{k}}$ and its neighbor nodes; $\mathrm{d}\left(\mathrm{S}_{\mathrm{k}}, \mathrm{S}_{\mathrm{j}}\right)$ is the Euclidean distance between node $\mathrm{S}_{\mathrm{k}}$ and $\mathrm{S}_{\mathrm{j}} ; \Gamma\left(\mathrm{L}_{\mathrm{kj}}, \alpha_{\mathrm{kj}}\right)$ is the function representing the relative positions of the neighbor nodes. Given the properties of the linear weighted Euclidean distance, there exists an optimal approximate solution $\min \Omega\left(S_{k}\right)$ for the related region of node $S_{k}$.

Mobile nodes used to repair the blind zone A must satisfy the following conditions: 
(1) Position of the mobile nodes: The overlapping region of the newly added mobile nodes and the original nodes must be smallest, while the area of the voids repaired is the largest.

(2) When determining the position of the mobile nodes, energy, distance and sensing radius of neighbor nodes along the boundaries of the blind zone must be considered.

(3) The number $\mathrm{k}$ of mobile nodes $\mathrm{k} \leq \frac{\sum_{-=1}^{\mathrm{n}} \Psi_{\mathrm{i}}}{\sum_{\mathrm{i}=1}^{\mathrm{n}} \Omega\left(\mathrm{S}_{\mathrm{i}}\right)} \Im\left(\eta\left(1-\mathrm{e}^{-\lambda \pi \mathrm{R}_{\mathrm{s}}^{2}}\right)\right)$

, where $\mathfrak{\Im}$ is the expectation function for the entire monitoring region.

(4) The coverage after introducing the mobile nodes must not be lower than the threshold coverage.

For any node $\mathrm{Si}$, the set of its related blind zone is $\left\{\Phi_{1} \ldots . . . \Phi_{\mathrm{m}}\right\}$. This set is composed of several nonoverlapping sub-zones $\Phi_{\mathrm{k}}$, where $\Phi_{\mathrm{k}} \mathrm{I} \Phi_{\mathrm{i}}=\varnothing$ and $\mathrm{k}=1$, 2 ...m. For any blind zone $\Phi_{k}$, the set of the static nodes along the boundaries of $\Phi_{\mathrm{k}}$ is $\mathrm{M}\{\mathrm{S} 1 \square \mathrm{Si}$...Sn $\}$. Node $\mathrm{M}$ constitutes a polygon $\overline{\Phi_{\mathrm{k}}}$. The Euclidean distance between two adjacent points on the boundaries of the polygon is $\mathrm{d}$ $\left(\mathrm{S}_{\mathrm{i}}, \mathrm{S}_{\mathrm{j}}\right)$. The approximate central point $\mathrm{H}$ of $\overline{\Phi_{\mathrm{k}}}$ is found by fuzzy decision making method. A closed triangular domain $\Gamma$ is formed by point $H$ and the sides of $\overline{\Phi_{k}}$. Weight $\kappa_{\mathrm{i}}$ of the sub-domain is $\Gamma_{\mathrm{i}}$

$$
\kappa_{\mathrm{i}}=\frac{\mathrm{F}\left(\mathrm{d}\left(\mathrm{S}_{\mathrm{i}}, \mathrm{S}_{\mathrm{j}}\right), \sum_{\mathrm{m}=\mathrm{i}}^{\mathrm{j}} \Im\left(\mathrm{Q}_{\mathrm{m}}, \mathrm{r}_{\mathrm{m}}\right)\right)}{\sum_{\mathrm{k}=1}^{\mathrm{n}} \Theta_{\mathrm{k}}}
$$

$\sum_{k=1}^{n} \Theta_{k}$ is the sum of residual energy of all nodes in the network; $\mathrm{F}$ is the weight function of the closed subdomain, correlated to the current residual energy $\mathrm{Q}$ of the static node and the sensing radius $r$ of the node. The more the closed triangular domain is divided in polygon $\overline{\Phi_{\mathrm{k}}}$, the more the boundary regions will be produced. The minimum weight $\bar{\zeta}_{i}$ of the closed triangular domain is given by

$$
\bar{\zeta}_{\mathrm{i}}=\arg \min \Gamma\left(\kappa_{1}, \kappa_{2} \ldots \ldots \kappa_{\mathrm{n}}\right)
$$

Closed triangular domain with the smallest weight is chosen. The side connecting static nodes $\mathrm{S}_{\mathrm{i}}$ and $\mathrm{S}_{\mathrm{j}}$ is $\mathrm{L}_{\mathrm{ij}}$. The mobile nodes must be found along the perpendicular bisector $\mathrm{C}_{\mathrm{ij}}$ of $\mathrm{L}_{\mathrm{ij}}$. The maximum sensing radius $\mathrm{R}_{\mathrm{m}}$ of the mobile node moves along $\mathrm{C}_{\mathrm{ij}}$. When and only when the length of arc formed by the sensing area intersecting with Lij is the smallest can we determine the optimal position of the first mobile node. With the mobile node deployed at this position, Fig. 3 shows the polygonal void $\overline{\Phi_{k}}$ is updated. Next another two adjacent points on the boundary of the polygon are selected to determine the position of the second mobile node. In the same manner, the mobile node $\mathrm{i}$ is deployed until the area of $\overline{\Phi_{\mathrm{k}}}$ is smaller than the threshold area S0 of the blind zone, that is, the blind zone for node $\mathrm{Si}$ is completely repaired. Thus all blind zones re-
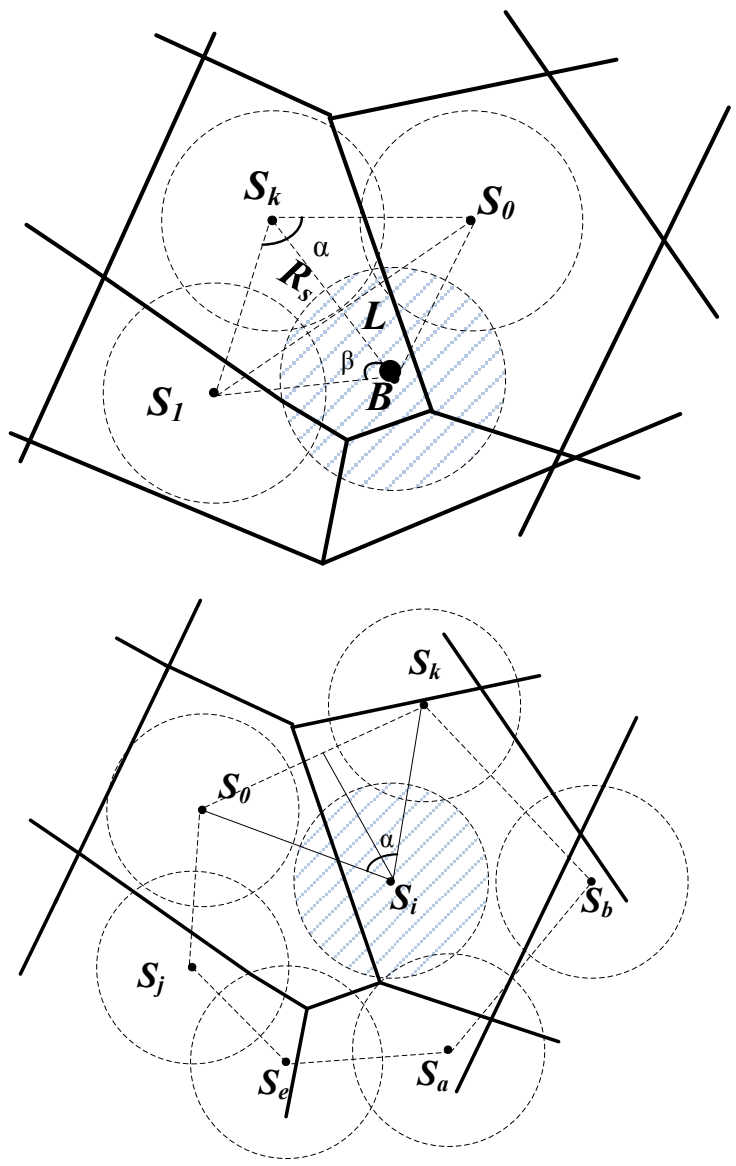

Figure 2. Schematic diagram of blind zones

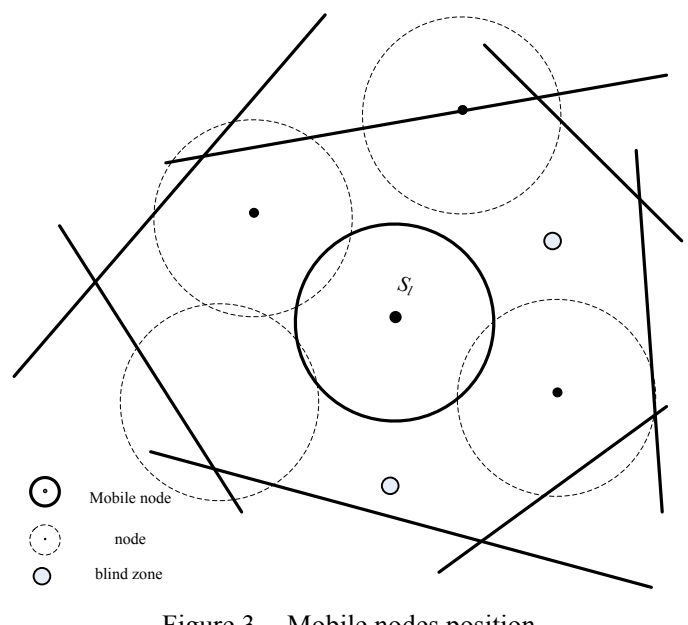

Figure 3. Mobile nodes position

lated to the non-overlapping static nodes in the network are repaired. The repair is finished when all static nodes in the network are traversed.

\section{SIMULATION EXPERIMENT}

Simulation experiment is carried out to compare the algorithm performance under the following scenario. For the monitoring region, 80 static nodes are deployed with sensing radius of $5-20 \mathrm{~m}$. The coverage optimization algorithm HPA in literature [7] is also implemented for comparison. The algorithm proposed in this paper is named as CMN. Performance indicators are coverage, network energy consumption and network lifetime. 
Coverage rate is the most important performance indicator of the network. For most scenarios, as long as the coverage is kept within a reasonable range, there will be no impact on network availability. Based on the sensing radius of static nodes, mobile nodes are introduced to reduce the blind zones and to increase the coverage. As shown in Fig. 4, at the initialization stage, the two algorithms have identical number and position of static nodes. At the early operation stage, the coverage of the two algorithms changes little. But with more mobile nodes introduced, CMN algorithm shows a slower deterioration of coverage as compared with HPA algorithm. Therefore, $\mathrm{CMH}$ algorithm outperforms HPA algorithm in coverage and convergence.

Energy consumption is another important consideration when evaluating the sensor network performance. Since the nodes vary in the amount of sensing tasks, the residual energy and sensing scope of the nodes will be different over time. $\mathrm{CMN}$ algorithm considers the factor of residual energy of the neighbor nodes in repairing the blind zones. This not only reduces the number of dead nodes, but also the probability of new blind zones due to dead zones. As shown in Fig. 5, both two algorithms show an increasing trend in energy consumption, but CMN algorithm has a lower energy consumption as compared with HPA algorithm.

Network lifetime is an intuitive performance indicator. After the network has reached a critical point of coverage, its performance will deteriorate and its service quality will decline until the end of network lifetime. As shown in Fig. 6 , the actual network service quality of CMN algorithm is slightly higher than expected and this algorithm has higher coverage and more balanced allocation of network resources than HPA algorithm. CMN algorithm is capable of determining the optimal position of the mobile nodes, so that the network coverage and connectivity are improved within the sensing radius of the nodes. As a result of this, network lifetime is prolonged.

\section{CONCLUSION}

Wireless sensor network is featured by a strong, dynamic variation, with nodes randomly deployed within the network. The operating nodes tend to show different residual energy after working for some time. Blind zones may appear, leading to incomplete monitoring data and impaired integrity of the network data transmitted and hence lower performance of the entire network. We propose a coverage optimization algorithm of wireless sensor network based on mobile nodes. The positions of the mobile nodes are determined with the consideration of residual energy of the static nodes, so that the risk of generating new blind zones due to energy exhaustion will decrease. The blind zones are repaired to the maximal extent using the proposed algorithm. Simulation experiment shows that the use of mobile nodes not only improves the coverage, but also prolongs the network lifetime and reduces the energy consumption. By keeping the number of nodes within a reasonable limit, the total cost is controlled as well.

\section{REFERENCES}

[1] Xing XF, Wang GJ, Li J. Polytype target coverage scheme for heterogeneous wireless sensor network using linear programming. Wireless Communications and Mobile Computing, 2014,14(8):1397-1408 http://dx.doi.org/10.1002/wcm.2269

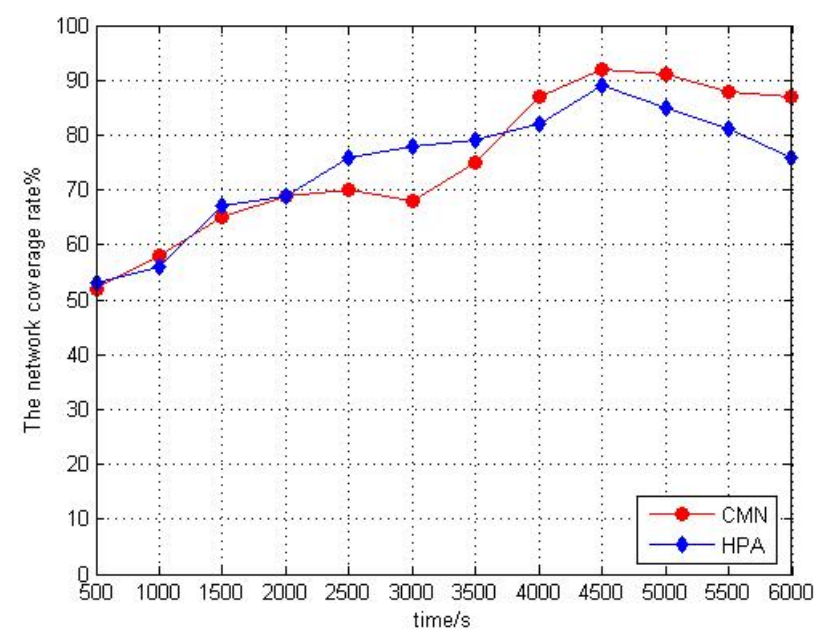

Figure 4. The network coverage rate

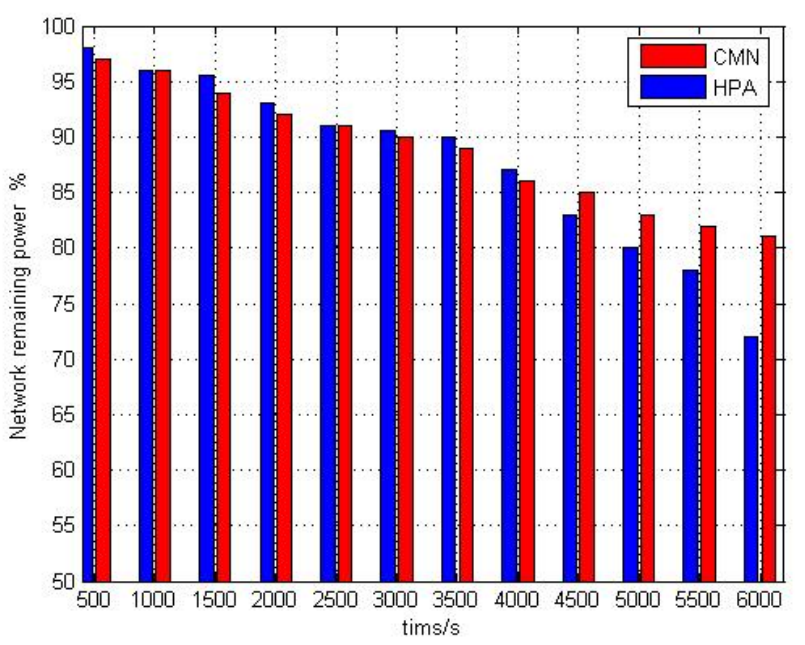

Figure 5. The network energy consumption

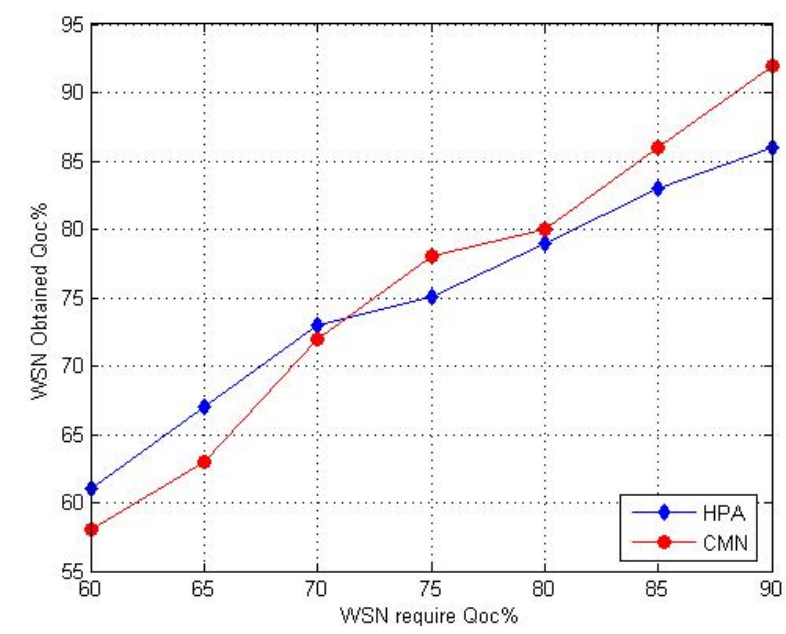

Figure 6. The network lifetime

[2] Sun ZY, Wu WG, Wang HZ, Chen H, Xing XF. A novel coverage algorithm based on event-probability-driven mechanism in wireless sensor network. EURASIP Journal on Wireless Communications and Networking, 2014,2014(1):1-17. http://dx.doi.org/10.1186/1687-1499-2014-58

[3] Wang LL, Wu XB. Decentralized detection and patching of trap coverage hole for sensor networks. Control and Decision, 2012, 27(12):1810-1815. 
[4] Tian D, Georganas ND. A node scheduling scheme for energy conservation in large wireless sensor networks. Wireless Communications and Mobile Computing, 2003,3(2):271-290. http://dx.doi.org/10.1002/wcm.116

[5] Bulusu N, Heidemann J, Estrin D A T T. Self-configuring localization systems: design and experimental evaluation[J] $\square \mathrm{ACM}$ Transactions on Embedded Computing Systems $\square 2004 \square 3(1)$ : 2460 http://dx.doi.org/10.1145/972627.972630

[6] Ye F, Zhong G, Lu SW, Zhang LX. A robust energy conserving protocol for long-lived sensor networks. Proc. of the 10th IEEE Int'l Conf. on Network Protocols. IEEE Computer Society, 2001. 200-201.

[7] X Li, DK Hunter, K Yang. Distributed Coordinate-Free Hole Recovery[A]. Proe IEEE Globecom[C]. New York: IEEE,2006.189-194.

[8] Wang W, Srinivasan V, Chua KC, Wang B. Energy efficient coverage for target detection in wireless sensor networks. In: Proc. Of the IPSN. Cambridge: ACM Press, 2007. 25-27. http://dx.doi.org/10.1145/1236360.1236401

[9] T. V. Chinh, and Y. Li, "Delaunay-triangulation based complete coverage in wireless sensor networks," IEEE PerCom, 2009.

[10] WF Cheng, Xk Liao, CX Shen. Maximal coverage scheduling in wireless directional sensor networks[J]. Journal of Software,2009,20(4):975-984. (Journal of Software)

[11] M Ma, YY Yang. Adaptive triangular deploymeng algorithm for unattended mobile sensor networks[J]. IEEE Transactions on Computers,2007,56(7):946-958. http://dx.doi.org/10.1109/TC.20 07.1054

[12] LIN J W, CHEN Y T. Improving the coverage of randomized scheduling in wireless sensor networks[J]. IEEE Transactions on Wireless Communications,2008,7(1):4807-4812.

[13] Wang B, Wang W, Srinivasan V, Chua KC. Information coverage for wireless sensor networks. IEEE Communications Letters,2005,9(11):967-969. http://dx.doi.org/10.1109/LCOMM.20 05.11002

[14] Erdelj M, Razafindralambo T, Simplot-Ryl D. Covering points of interest with mobile sensors. IEEE Trans. on Parallel and Distributed Systems, 2013,24(1):32-43. http://dx.doi.org/10.1109/TP DS.2012.46

[15] Dinesh D, Arobinda G, Arijit B, Subhas C. Line coverage measures in wireless sensor networks. Journal of Parallel \& Distributed Computing, 2014,24(24):2596-2614.

[16] He SB, Chen JM, Sun YX. Coverage and connectivity in dutycycled wireless sensor networks for event monitoring. IEEE Trans. on Parallel and Distributed Systems, 2012,23(3):475-482. http://dx.doi.org/10.1109/TPDS.2011.191
[17] Y YOON, Y H KIM. An efficient genetic algorithm for maximum coverage deployment in sensor networks[J]. IEEE Transaction on Cybernetics,2013,43(5):2168-2267.

[18] Hu X M,Zhang J,et al.Hybrid genetic algorithm using a forward encoding scheme for lifetime maximization of wireless sensor network[J].IEEE Trans Evol Comput, 2010, 14(5): 766-781. http://dx.doi.org/10.1109/TEVC.2010.2040182

\section{AUTHORS}

Li Zhu female, born in 1982, received her M.S. degree in Computer Applied Technology from University of Electronic Science and Technology of China (2011). Now she is reading for a doctor degree at Beijing University of Posts and Telecommunications. Her research interests include WSN and embedded system. (Email: jolie.zhl@hotmail.com / zhul@nercita.org.cn)

Chunxiao Fan female, born in 1962, she is currently a professor and the director of Center for Electronic and intelligent systems. Meanwhile, she is a members of the national sensor network working group. Her research interests include WSN, data mining and intelligent information processing, etc. (Email: cxfan@bupt.edu.cn)

Huarui $\mathbf{W u}$ is a professor at National Engineering Research Center for Information Technology in Agriculture. $\mathrm{He}$ is interested in studying Artificial Intelligence. In recent years, he has participated in 18 national and provincial key scientific research projects, and published over 20 academic papers. He got the first prize of Beijing Science and Technology in 2005, and third prize of agricultural technology promotion in 2003. (Email: wuhr@nercita.org.cn)

Zhigang Wen is a professor at Beijing University of Posts and Telecommunications, meanwhile, he is a members of the national sensor network working group. He research interests include WSN, Cognitive radio, the system communication, etc. (Email: zwen@bupt.edu.cn)

The work presented in this paper was supported by the National Natural Science Foundation of China (Grants No. NSFC- 61471067), the National Natural Science Foundation of China (Grants No. NSFC61271257) and the National Natural Science Foundation of China (Grants No. NSFC- 61571051) Submitted 22 July 2016. Published as resubmitted by the authors 25 August 2016. 